\title{
Context and Teaching Chinese as a Foreign Language*
}

\author{
${ }^{*}$ Xiao Jiugen ${ }^{1}$ Chen Xijuan ${ }^{2}$ Wang Jun ${ }^{3}$ \\ 1. *Jiangxi Normal University, College of Liberal Arts, Nanchang Jiangxi 330022; \\ 2 Nanchang Institute of Science and Technology, Education Department for Ethnic Minorities, \\ Nanchang Jiangxi 330108) \\ 3. Jiangxi Normal University, College of Liberal Arts, Nanchang Jiangxi 330022. \\ *jxsdxjg666666@sina.com, chenxijuan1985@163.com, jxsdjunw@126.com.
}

Keywords: Context; Language learning; Teaching Chinese as a foreign language

\begin{abstract}
Context plays an important role in linguistic theory, and the understanding and use of language cannot do without specific context. Through the elaboration of context in verbal knowledge and its application in teaching Chinese as a foreign language, this paper puts forward the basic way of context teaching, and emphasizes the importance of context in foreign language such as spoken language, grammar, practice teaching, for the research on language learning and teaching Chinese as a foreign language.
\end{abstract}

\section{Introduction}

With the rapid development of our economy and the continuous improvement of international status, more and more people overseas come to China to learn Chinese. Many people spent a lot of time learning Chinese words and grammar for a better communication, but very few people can accurately, smoothly and freely use Chinese to communicate. Because mastering a language requires students not only master the pronunciation, vocabulary, semantics and grammar, but also can appropriately and flexibly express in a specific context, which is the ultimate goal of teaching Chinese as a foreign language.

Zhang Zhigong, a renowned linguist, educator in China, said, "Language is always used in a concrete communication environment. Therefore, the analysis of language must rely on the language context, without a certain context, it is difficult to determine the structure and meaning of the language with the isolated fragments." ${ }^{[1]}$ The "context" here refers to of language communicative environment. In teaching Chinese as a foreign language, it has been noted that context plays an important accurately role in learning Chinese for students to grasp it accurately, and also people take how to improve the students' ability of using language and comprehension ability as one of the most important purpose of teaching Chinese as a foreign language. Therefore, in teaching Chinese as a foreign language, it is very important to properly introduce the theory of context.

\section{Elements and the importance of context}

The context elements. Since this notion of context was proposed by British anthropologists B.Malinowski in 1923, many foreign linguistics and scholars have given their interpretation about it

\footnotetext{
"Brief introduction of author: Xiao Jiugen (1960-)male, PH.D in Literature, vice professor in Jiangxi Normal University, Master's Supervisor, Committee in Jiangxi Association of Chinese Linguistics, the council member in National Modern Chinese Teaching and Researching Association, engaging in the research on social linguistics, completing many provincial and ministerial research projects on social science planning funds, participating in the project " 'Project 985'Cutural Legacy and Culture Exchange" with innovation funds and social science planning funds, publishing over 30 academic articles in national journals.
} 
from different aspects, which is conducive to the enrichment and development of the theory of context.

In 1957, Firth pointed out that context consists of two parts, namely context constituted by language factors and situational context constituted by non-language factors. In 1964, the British functional school linguist Hallidy proposed the term "register", which means the content is context.

Chinese scholars Mr. Chen Wangdao firstly put forward the concept "context". He noted six factors of context, "why", "what", "who", "where", "when", "what".

With the deepening of the study on context theory, scholars have put forward many original ideas. Context is a dynamic and open system, the subjective factors are closely related to the objective factors in the process of verbal communication.

In view of the current research results, people think that the context is the environment depending on the verbal communication, which is understood by the both communicative sides, and is the element ensuring the smooth conduct of the communication, and it includes various factors affecting discourse issued and understanding, including not only the elements inside and outside of the language of speech, also the cognitive factors of both sides in the communication. ${ }^{[2]}$

It is generally believed that, the elements of the context can be divided in both narrow sense and broad sense. In narrow sense, the elements are formed by the language environment both in written form and spoken form; while the generalized elements in broad sense refer to the specific environment of the expressions (not only referring to specific occasions, but also referring to the social environment). That is to say, the content of the context is very rich, including:

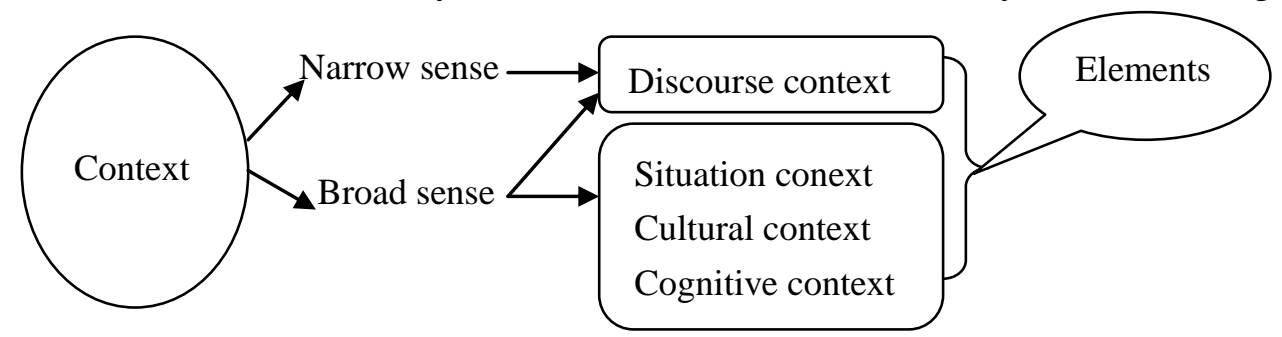

Figure. 1

The importance of context. Context plays an important role in the use and understanding of speech. On the function of context, the former scholars have done a lot of research. A Japanese scholar, Xizhi Guangzheng put forward eight functions for the context: absolute function, restrictive function, explaining function, design function, filtering and filling function, generating function, transforming function, acquisition function. He believes that the restrictive function of context is the influence of context on language use and research. ${ }^{[3]} \mathrm{Wu}$ Tieping thinks that context has two functions, one is that speech segments dependent on context, the other is that the context restricts speech segments ${ }^{[4]}$; Jin Dingyuan points out that context is with two functions, explanation and the filtration, ${ }^{[3]}$ etc. At present, the common view of scholars on the basic functions of context is that, context is restrictive and interpretive. The so-called restrictive function, on the one hand, refers to the restriction generated in the discourse, namely the constraints on the speaker using language in speech communication, which requires the speaker to select the proper language expression being consistent with communicative situations, namely the choice of words is restricted by the context, so different time, places, people require different selection for the mode of expression; on the other hand, the hearer in discourse understanding should combine with the specific context to effectively understand the utterance. The explanation function is based on the listener, since the context focuses on a specific and concrete meaning, thus contributes to the interpretation and explanation of some language phenomena, and can effectively eliminate the ambiguity and polysemy in the 
interpretation of words. ${ }^{[5]}$ It can be shown as follows:

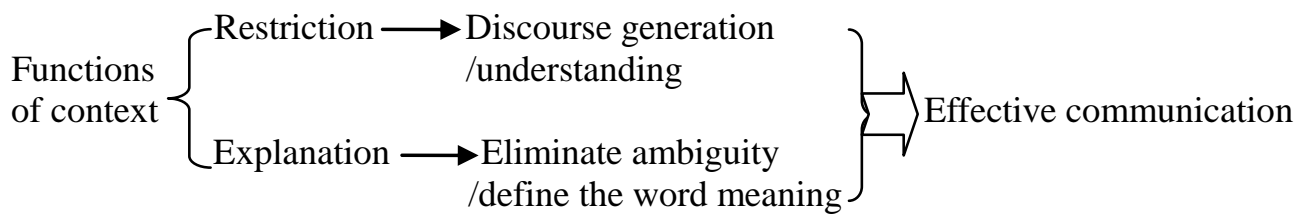

Figure. 2

\section{Basic approaches to teaching in context}

What is the relationship between context and language teaching? How to combine the context with language teaching or how to implement the context teaching in language teaching? Those problems are worth to discuss.

As everyone knows, language teaching cannot do without a specific context. Therefore, in the process of teaching, teachers must consider a variety of contexts, both its narrow context and generalized context.

So, in the process of language teaching, what should people do in the context teaching? It can be shown as follows,

First of all, students are required to understand the semantics and discourse context in the context teaching. Generally speaking, the understanding of discourse is based on the context. The hearer makes the reasoning on the above sentences, and then further explains them, which become the basis of hearer's understanding of the speaker's intention. ${ }^{[6]}$ The discourse context is composed by the sentence or a group of sentences, or by a variety of forms such as paragraphs or chapters. In the process of language using, the meaning of a word often changes in the different context, and the understanding of the meaning of a word depending on the concrete context will change. For example:

Grandpa Zhang often calls me "little thing", "little devil", "bad thing". (Chen Zhuo Practical Chinese Intermediate Tutorial (the first edition), Beijing Language Press, 2006, pp. 11-12)

The word "thing" generally refers to the object, when use "thing" to refer to a person, the situation is limited, generally neither used for affirmative, nor used in negative sentences and questions, such as, people cannot say like this, "She is a thing", or "she is not a thing" (containing the curse meaning). The specific meaning of the above example is shown with the context. Grandpa Zhang made fun of me, so the concrete meaning of the words means he loves me.

Thus, a language word often has more than one meaning, different context with different meanings. Therefore, learning words in the concrete context plays a multiplier effect in study.

Secondly, the situational context teaching is applied to cultivate the student's communicative competence. Situational context refers to the context directly relating to the communicative field, but constricted by the particular speech situation, such as time, place, things, etc. The old saying "the same words to the same kind of people." shows that the appropriate mode of expression should be selected in different communication situations. Teachers should pay attention to different expressions to different occasions when teaching, also should cultivate students' ability to recognize various situations, by setting the communication situation in teaching, and encouraging students to participate in the language practice activities. To improve the students' adaptability of language and culture, teachers must combine the language teaching with in the specific communicative situation, making the students realize that language usage is closely related to the context.

Thirdly, the cultural context teaching is applied to cultivate the student's appropriate speech ability. Language is the carrier of culture, so the deep understanding of a language requires the 
understanding of the culture. Such as learning Chinese, is essentially related to understand differences in communication.

Finally, the cognitive context teaching is applied to cultivate students the ability of language construction. Language users through experience or thinking make the specific context internalized and cognitive, the result of the internalization and cognition is the cognitive context in the brain, people in communication often rely on the cognitive context. ${ }^{[8]}$

For the cognitive context, teachers should first fully understand the students' knowledge of the language that they have grasped, and combine the new knowledge for the students with the old knowledge together on the basis of their original knowledge structure, thus forming a knowledge network, which can make the students blend and grasp all the knowledge. In addition, more familiar with the students' cultural background is required for the teachers, being familiar with the reality of their living situation, on the basis of the situation, teachers can design some teaching content and topic closely related to students' daily life, which can not only improve their interest in learning, but more important enhance their confidence to express. ${ }^{[9]}$

In a word, in the process of teaching Chinese as a foreign language, teachers should have a full understanding of the contextual restriction on Chinese learning and teaching, and give full play to the functions of the context of positive influence on Chinese teaching. By using the comparative, deductive and inductive methods, teachers consciously apply the context theory mode of operation in teaching students, so that students can use the context theory to guide language learning. ${ }^{[10]}$

At present, although more and more people pay attention to the context teaching in teaching Chinese as a foreign language, in the actual classroom teaching, some teachers still do not jump out of the old teaching mode, and can be unable to create a new mode of teaching from the perspective of context.

Although a lot of researches have been done on context, most of the researches focus on the context function on language understanding and learning, and few for the teaching in a concrete class to discuss how to combine the study of context elements with teaching. It undoubtedly should arouse the attention of people.

Chinese culture. Cultural background is very important in language teaching, which is formed by a nation's unique cultural traditions and customs in the long process of historical development. For example, in teaching Chinese as a foreign language, one of the students tells us that Burmese see crow as a holly bird, called the "God crow", while Chinese don't like crow, such as the proverb "the crows around the world are all black".

Thus, cultural differences can affect and even mislead language information acquire, can also cause communication barriers. Research on the cultural factors in the context is conducive to the cross-cultural language communication. ${ }^{[7]}$ So, teachers should consciously strengthen the cultural research in language and teaching, so that students will deepen the understanding and mastery of the content under the culture background, thereby reducing the obstacles or misunderstanding caused by cultural

\section{The organisc combination of the context and teaching chinese as a foreign language}

The study of context has the important significance to the study of language, but also for language teaching, especially in teaching Chinese as a foreign language with more practical value. In teaching Chinese as a foreign language, if people neglect this important factor of context, the students even have mastered some basic knowledge, commonly used sentences in Chinese, in a specific context, they still can not master appropriate, flexible use rule of Chinese, and thus it will be difficult for them to achieve the expected effect of communication. 
In recent years, with the development of language teaching, context, in some new teaching methods such as functional teaching, situational teaching, audio-visual teaching, has been used widely, and it has influenced or are influenced the teaching Chinese as a foreign language. ${ }^{[11]}$

How to organically combine context with teaching Chinese as a foreign language? People believe that teachers should carry out teaching pattern according to the specific context in the teaching process of Chinese spoken language, grammar and practice classes.

The context and the teaching of Chinese as a foreign language. The teaching aim of oral English class is to improve the students' ability of oral expression and social ability, and context is particularly important in the situation. Such as the learning of the topic "directions", first, try to be familiar with the surrounding and find the map, teachers and students or between students can conduct relative real exchange, such as, where is the office building, where is the library, how to find a supermarket and so on, through the context teaching, students can be impressed for the questions and answers, which can receive double teaching effect.

In communicative activities, people express his intentions by words, but sometimes people's intention do not just equal to what its literal meaning, especially limited by different cultural background even with the same speech act, the understanding and use of the words also have very big difference. For example, many students do not know when to say "have you eaten?" in an appropriate way, because in western countries, if you ask a person to eat implies that you want to invite him to have a meal, while in Chinese, it is just for greeting.

Verbal communication cannot do without the specific communicative context. Different conversation topics, different communicative situations, different conversation atmosphere all constitute the special communicative context. First of all, teachers should encourage students to stimulate their interest in the communicative activities, then remind students of the context elements like, time, place, object in the oral communication. ${ }^{[12]}$

The context and the teaching of Chinese grammar. Lv Shuxiang once said, "the meaning any words in any language, do not equal to the sum of the meaning of the words, but more that. According to the math theory, two plus two equals four, not five. The language does not like that." "The meaning in people's language is quite complex, including the significance of the language itself and the effect of language environment." "So it is said like that, 'The meaning is implied', 'the real meaning is beyond the literal meaning'." ${ }^{[13]}$ For example, in the sentence "it is going to rain", the literal meaning refers to the raining will happen, but the specific meaning depends on the different contexts:

(1) On the way home, the mother says to her daughter: "it's going to rain." The sentence refers to hurry up and come back home.

(2)When you go out, the mother says to her daughter: "it's going to rain." The sentence refers to take an umbrella with you.

(3) In the hot summer, the mother says to her daughter: "it's going to rain." The sentence refers to the weather is finally going to be cool.

In Chinese grammar teaching, the context holds a very great proportion, and many grammatical points require the teacher's blackboard writing and context description. The grammar item setting is the representation of context, which requires teachers to summarize these grammar items appeared in the specific pragmatic environment.

At present, in the grammar teaching, teachers still focus on the grammar knowledge with isolated analysis on some grammar rules, ignoring the specific context and practical application. In fact, some difficult grammar teaching for both teachers and students can be easy to be solved in the specific context. ${ }^{[14]}$ 
The context and foreign language teaching practice. The language practice teaching of the activities with a purpose and a plan can more effectively for Chinese learners to create good language context. Students are required to go out of the classroom, out of books, out of the campus, to the rich and colorful world, which is an excellent place for them to experience life and learn language. To improve the efficiency of class teaching, teaches should encourage the students to practice from passive to active practice. Social practice can eliminate some untrue language material without applicable or applicable value in the grammar teaching, students can contact the real application in language. Students are required to gain a better understanding of our nation's social culture through practice, experience, which can help them quickly and correctly learn to use Chinese to make Chinese communicative activities.

The figure is shown as follows:

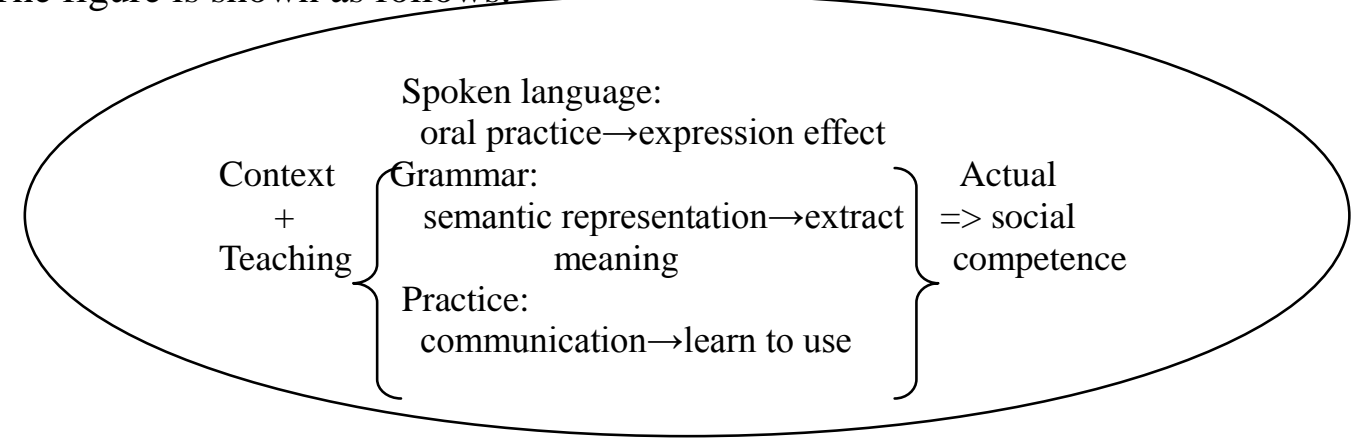

Figure. 3

\section{Conclusion}

The famous linguist Ferdinand de Saussure said, "The value of any word is determined by its surrounding context, if not first inspect its context, even the word value cannot be sure." ${ }^{[15]}$ Context plays an important role in the understanding and use of language. When people use language to communicate, the meanings of language expressions are very rich and complicated, not only understanding from the lexical meaning and grammatical meaning, but depending on the context. Only in this way can people in speech communication to eliminate vagueness and ambiguity, and achieve the accurate understanding of the purpose of the language.

Emphasizing the important role of context in teaching Chinese as a foreign language, means to encourage teachers to use the real communicative situation as far as possible in the teaching process, so that the students can directly perceive language in the social environment, which can not only enable them to learn the language faster, but also improve the ability of language expression.

Therefore, in order to achieve good teaching effect in teaching Chinese as a foreign language, teachers need to use the context teaching method, through the spoken teaching, grammar teaching and social practice to simulate and create the scene, and students are required to enter into the language environment to know Chinese, learn Chinese, as well as use Chinese.

\section{Reference}

[1] Zhang Zhigong. Modern Chinese [M]. People's Education Press, 1982

[2] Li Yingfei. Functions of Context in Teaching Chinese as a Foreign Language [J]. Journal of Shanghai International Studies University, 2007(6) 
[3] Xizhi Guangzheng (Janpan). Papers on Context [M]. Beijing Language Institute Press, 1992

[4] Wu Tieping. General linguistic Summary [M]. Higher Education Press, 2006

[5] Zhang Lige. Context and Verbal Communication [J]. Foreign Language Teaching and Learning, 2001(3)

[6] Wang Fenglan. Pragmatic Competence, Context and Teaching Chinese as a Foreign Language Education [J]. Contemporary Education Science, 2003(12)

[7] Chen Jie. Study on Pragmatic Competence and Context and Teaching Chinese as a Foreign Language [J]. 2009 (16)

[8] Li Shengping. Context and Teaching Chinese as a Foreign Language [J]. Overseas Chinese Education, 2009 (4)

[9] Zhou Mingqiang. Study on the Context Strategy in Chinese Teaching [J]. Journal of Zhejiang College of Education, 2003 (2)

[10] Sun Lan. Analysis on Context Teaching in Teaching Chinese as a Foreign Language [J]. Journal of Yunnan Normal University 2009 (4).

[11] Chang Jingyu. Pragmatic, Semantic, Grammar [M]. Hangzhou University Press, 1996

[12] Lou Xiurong. Context and Teaching Chinese as a Foreign Language [J]. Journal of Shenyang Teachers College, 1997 (3)

[13] Lv Shuxiang. Study on Chinese [M]. Life·Reading.Konwledge San Lian Bookshop Press, 1980

[14] Wang Wei. The Function of Context on Teaching Chinese as a Foreign Language [J]. Social Sciences in Jiangsu,.2000 (6)

[15] Ferdinand de Saussure (Swiss). General Linguistics [M]. London Press, 1960 